Pacific Journal of Mathematics

TWO INEQUALITIES IN NONNEGATIVE SYMMETRIC 


\section{TWO INEQUALITIES IN NONNEGATIVE SYMMETRIC MATRICES}

\section{DAVID LONDON}

Marcus and Newman have made the following conjecture: Let $A=\left(a_{i j}\right)$ be a $n \times n$ nonnegative symmetric matrix. Then

$$
S(A) S\left(A^{2}\right) \leqq n S\left(A^{3}\right) \text {, }
$$

where

$$
S(A)=\sum_{i, j=1}^{n} a_{i j}
$$

After reducing the conjecture to a standard maximum problem of linear programming we prove that it holds for $n \leqq 3$. A counter example shows that for $n \geqq 4$ the conjecture is wrong.

We also consider the following conjecture: Let $A=\left(a_{i j}\right)$ be a $n \times n$ nonnegative symmetric matrix. Then

$$
S\left(A^{m}\right) \leqq \sum_{i=1}^{n} s_{i}^{m}, \quad m=1,2, \cdots,
$$

where

$$
s_{i}=\sum_{j=1}^{n} a_{i j}, \quad i=1, \cdots, n .
$$

The validity of this conjecture is established in two cases: (1) $m$ up to 5 and any $n$, (2) $n$ up to 3 and any $m$. The general case remains open. We conclude this paper with two generalizations of the second theorem.

Notation. Let $A=\left(a_{i j}\right)$ be a $n \times n$ real matrix. $A$ is called nonnegative if $a_{i j} \geqq 0, i, j=1, \cdots, n$. The quadratic form corresponding to a symmetric $A$ is denoted by $A(x, x)$, that is

$$
A(x, x)=(A x, x)=\sum_{i, j=1}^{n} a_{i j} x_{i} x_{j}
$$

Here $(A x, x)$ denotes, as usually, the scalar product of the real vectors $x$ and $A x$. Denote $e=(1, \cdots, 1)$ and $A e=\left(s_{1}, \cdots, s_{n}\right)=s=s(A)$. $s_{i}=s_{i}(A)$ is thus the sum of the elements of the $i$ th row of $A$. $s=s(A)$ is the row sums vector of $A$. $A$ is generalized stochastic if $A$ is nonnegative and if $s(A)=c e$, where $c$ is a scalar. Further

Received October 8, 1964. This paper represents part of a thesis submitted to the Senate of the Technion-Israel Institute of Technology in partial fulfillment of the requirements for the degree of Doctor of Science. 
notations are:

$$
\begin{aligned}
S(A) & =\sum_{i, j=1}^{n} a_{i j}=A(e, e), \\
S(x) & =\sum_{i=1}^{n} x_{i}, \quad x=\left(x_{1}, \cdots, x_{n}\right), \\
A^{m} & =\left(a_{i j}^{(m)}\right), \\
s_{i}^{(m)} & =s_{i}^{(m)}(A)=s_{i}\left(A^{m}\right)=\sum_{j=1}^{n} a_{i j}^{(m)}, \\
s^{(m)} & =s^{(m)}(A)=s\left(A^{m}\right) .
\end{aligned} \quad m=1,2, \cdots .
$$

1. The conjecture of Marcus and Newman.

1.1. The conjecture and its connection with linear programming. In [4, p. 634] the following conjecture is introduced: Let $A=\left(a_{i j}\right)$ be $a n \times n$ nonnegative symmetric matrix. Then

$$
S(A) S\left(A^{2}\right) \leqq n S\left(A^{3}\right) \text {. }
$$

Using the notation introduced before, we have

$$
\left\{\begin{array}{l}
S(A)=\sum_{i=1}^{n} s_{i}, \\
S\left(A^{2}\right)=\sum_{i=1}^{n} s_{i}^{(2)}=A^{2}(e, e)=(A e, A e)=\sum_{i=1}^{n} s_{i}^{2}, \\
S\left(A^{3}\right)=\sum_{i=1}^{n} s_{i}^{(3)}=A^{3}(e, e)=\left(A e, A^{2} e\right)=\sum_{i=1}^{n} s_{i} s_{i}^{(2)} .
\end{array}\right.
$$

Hence, (1.1) can be written in the form

$$
n \sum_{i=1}^{n} s_{i} s_{i}^{(2)}-\sum_{i=1}^{n} s_{i} \sum_{i=1}^{n} s_{i}^{(2)} \geqq 0 \text {. }
$$

If the sets $s=\left(s_{1}, \cdots, s_{n}\right)$ and $s^{(2)}=\left(s_{1}^{(2)}, \cdots, s_{n}^{(2)}\right)$ are similarly ordered, that is if $\left(s_{i}-s_{j}\right)\left(s_{i}^{(2)}-s_{j}^{(2)}\right) \geqq 0$ for every $1 \leqq i, j \leqq n$, then according to an inequality of Tchebychef [2, p. 43] the inequality (1.3) holds. However, the following example shows that for nonnegative symmetric matrices $A, s(A)$ and $s^{(2)}(A)$ need not be similarly ordered. Let

$$
A=\left[\begin{array}{lll}
6 & 2 & 0 \\
2 & 1 & 0 \\
0 & 0 & 4
\end{array}\right] .
$$

Then $s(A)=(8,3,4)$ and $s^{(2)}(A)=(54,19,16) . \quad s(A)$ and $s^{(2)}(A)$ are therefore not similarly ordered.

Denote

$$
\sum_{j=1}^{n} a_{i j}=\sum_{j=1}^{n} a_{i j}-a_{i i}
$$


We have

$$
\begin{array}{ll}
a_{i i}=s_{i}-\sum_{j=1}^{n} a_{i j}, & i=1, \cdots, n, \\
s_{i}^{(2)}=\sum_{j=1}^{n} a_{i j} s_{j}, & i=1, \cdots, n .
\end{array}
$$

From (1.2), (1.4) and (1.5) follows

$$
\begin{aligned}
n S\left(A^{3}\right) & -S(A) S\left(A^{2}\right)=n \sum_{i=1}^{n} s_{i}^{(2)} s_{i}-\sum_{i=1}^{n} s_{i} \sum_{i=1}^{n} s_{i}^{(2)} \\
= & n \sum_{i=1}^{n} s_{i}\left[\sum_{j=1}^{n} a_{i j} s_{j}+s_{i}\left(s_{i}-\sum_{j=1}^{n} a_{i j}\right)\right]-\sum_{i=1}^{n} s_{i} \sum_{i=1}^{n} s_{i}^{2} \\
= & n \sum_{i=1}^{n} s_{i}^{3}-\sum_{i=1}^{n} s_{i} \sum_{i=1}^{n} s_{i}^{2}-n \sum_{1 \leqq i<j}^{n} a_{i j}\left(s_{i}-s_{j}\right)^{2} \\
= & \sum_{1 \leq i<j}^{n}\left(s_{i}-s_{j}\right)\left(s_{i}^{2}-s_{j}^{2}\right)-n \sum_{1 \leqq i<j}^{n} a_{i j}\left(s_{i}-s_{j}\right)^{2} .
\end{aligned}
$$

Hence,

$$
\begin{aligned}
n S\left(A^{3}\right) & -S(A) S\left(A^{2}\right) \\
& =\sum_{1 \leqq i<j}^{n}\left(s_{i}+s_{j}\right)\left(s_{i}-s_{j}\right)^{2}-n \sum_{1 \leqq i<j}^{n} a_{i j}\left(s_{i}-s_{j}\right)^{2} .
\end{aligned}
$$

Using (1.6) we obtain. a representation of the conjecture (1.1) by concepts of linear programming (see e.g. Gale [1]). Consider the following maximum problem: Let $s_{1}, \cdots, s_{n}$ be nonnegative numbers. Find numbers $a_{i j}=a_{j i}, i \neq j ; i, j=1, \cdots, n$, which satisfy the set of linear inequalities

$$
\left\{\begin{aligned}
a_{i j}=a_{j i} \geqq 0, i \neq j ; & i, j & =1, \cdots, n, \\
\sum_{j=1}^{n} a_{i j} \leqq s_{i}, & i & =1, \cdots, n,
\end{aligned}\right.
$$

and which maximize the linear function

$$
\sum_{1 \leqq i<j}^{n} a_{i j}\left(s_{i}-s_{j}\right)^{2}
$$

The problem (1.7), (1.8) is a maximum standard problem of linear programming. A set of numbers $a_{i j}$ which satisfies the inequalities (1.7) is a feasible solution of the problem. A feasible solution which maximizes (1.8) is an optimal solution. The dual of the problem (1.7), (1.8) is the following minimum standard problem: Find numbers $y_{1}, \cdots, y_{n}$ which satisfy the set of inequalities

$$
\left\{\begin{array}{l}
y_{i} \geqq 0, i=1, \cdots, n, \\
y_{i}+y_{j} \geqq\left(s_{i}-s_{j}\right)^{2}, i \neq j ; \quad i, j=1, \cdots, n,
\end{array}\right.
$$


and which minimize the function

$$
\sum_{i=1}^{n} s_{i} y_{i}
$$

It is obvious that the problem (1.7), (1.8) and its dual have optimal solutions.

From (1.6) it follows that the conjecture (1.1) can be represented in the following equivalent form: Let $\tilde{a}_{i j}, i \neq j ; i, j=1, \cdots, n$, be an optimal solution of the maximum standard problem (1.7), (1.8). Then

$$
\sum_{1 \leqq i<j}^{n} \tilde{a}_{i j}\left(s_{i}-s_{j}\right)^{2} \leqq \frac{1}{n} \sum_{1 \leq i<j}^{n}\left(s_{i}+s_{j}\right)\left(s_{i}-s_{j}\right)^{2} .
$$

1.2. Proof for $n \leqq 3$. In this section we establish the validity of the conjecture for $n \leqq 3$.

THEOREM 1. Let $A$ be $a n \times n$ nonnegative symmetric matrix. Then for $n \leqq 3$ the inequality (1.1) holds. The equality sign holds in (1.1) if and only if $A$ or $A^{2}$ is a generalized stochastic matrix.

Proof. For $n=1$ the inequality (1.1) holds trivially. For $n=$ 2,3 we use the representation of (1.1) by (1.9).

For $n=2$ it is sufficient to prove that if

$$
0 \leqq a_{12} \leqq \min \left(s_{1}, s_{2}\right) \text {, }
$$

then

$$
a_{12}\left(s_{1}-s_{2}\right)^{2} \leqq \frac{1}{2}\left(s_{1}+s_{2}\right)\left(s_{1}-s_{2}\right)^{2}
$$

(1.10) implies

$$
a_{12} \leqq \frac{s_{1}+s_{2}}{2},
$$

and from (1.12) follows (1.11). Equality holds in (1.1) if and only if it holds in (1.11), and there it holds if and only if $s_{1}=s_{2}$, that is if $A$ is a generalized stochastic matrix. As by (1.3) we clearly have equality in (1.1) if $A^{2}$ is generalized stochastic, it follows that there are not nonnegative symmetric $2 \times 2$ matrices such that $A^{2}$ but not $A$ is generalized stochastic. We remark that it is easily seen that for $n=2, s$ and $s^{(2)}$ are similarly ordered sets. (1.1) thus follows also from the inequality of Tchebychef. ${ }^{1}$

1 As the referee suggests, the proof for $n=2$ can be done directly by the methods in [4]. Using the notations in [4], we have

$$
2 S\left(A^{3}\right)-S(A) S\left(A^{2}\right)=w_{1} w_{2}\left(\lambda_{1}-\lambda_{2}\right)^{2}\left(\lambda_{1}+\lambda_{2}\right) \text { and } \lambda_{1}+\lambda_{2}=\operatorname{tr}(A) \geqq 0 .
$$

The author wishes to thank the referee for this remark. 
We prove now the theorem for $n=3$. Without loss of generality we may assume that

$$
0<s_{1} \leqq s_{2} \leqq s_{3} .
$$

The assumption $0<s_{1}$ does not restrict the generality. If $s_{1}=0$ then $A$ is of the form

$$
A=\left[\begin{array}{lll}
0 & 0 & 0 \\
0 & B & \\
0 & &
\end{array}\right] .
$$

Hence, using the validity of (1.1) for $n=2$, we obtain

$$
S(A) S\left(A^{2}\right) \leqq 2 S\left(A^{3}\right) \text {. }
$$

At first we treat the case

$$
0<s_{1}<s_{2}<s_{3} .
$$

Denote

$$
a_{23}=a_{32}=x_{1}, \quad a_{13}=a_{31}=x_{2}, \quad a_{12}=a_{21}=x_{3} .
$$

The corresponding maximum problem is: Maximize

$$
M\left(x_{1}, x_{2}, x_{3}\right)=x_{1}\left(s_{2}-s_{3}\right)^{2}+x_{2}\left(s_{1}-s_{3}\right)^{2}+x_{3}\left(s_{1}-s_{2}\right)^{2},
$$

where $x_{i} \geqq 0, i=1,2,3$, satisfy the system of inequalities

$$
\begin{cases}(1) & x_{2}+x_{3} \leqq s_{1} \\ \text { (2) } & x_{1}+x_{3} \leqq s_{2} \\ \text { (3) } & x_{1}+x_{2} \leqq s_{3} .\end{cases}
$$

The dual of the problem (1.15), (1.16) is the following problem: Minimize

$$
y_{1} s_{1}+y_{2} s_{2}+y_{3} s_{3}
$$

where $y_{i} \geqq 0, i=1,2,3$, satisfy the system of inequalities

$$
\left\{\begin{array}{l}
y_{2}+y_{3} \geqq\left(s_{2}-s_{3}\right)^{2} \\
y_{1}+y_{3} \geqq\left(s_{1}-s_{3}\right)^{2} \\
y_{1}+y_{2} \geqq\left(s_{1}-s_{2}\right)^{2}
\end{array}\right.
$$

Let $\widetilde{x}_{1}, \widetilde{x}_{2}, \widetilde{x}_{3}$ be an optimal solution of $(1.15)$, (1.16) and $\widetilde{y}_{1}, \widetilde{y}_{2}, \widetilde{y}_{3}$ an optimal solution of the dual problem. Let $(\widetilde{1.16}),\left(\widetilde{1.16^{\prime}}\right)$ denote respectively the inequalities (1.16), (1.16') after substituting $\widetilde{x}_{1}, \widetilde{x}_{2}, \widetilde{x}_{3}$ and $\widetilde{y}_{1}, \widetilde{y}_{2}, \widetilde{y}_{3}$ respectively.

According to our assumption (1.14) we have 


$$
\left(s_{i}-s_{j}\right)^{2}>0, i \neq j ; i, j=1,2,3,
$$

and it follows therefore from $(\widetilde{1.16})$ that at most one of the numbers $\widetilde{y}_{1}, \widetilde{y}_{2}, \widetilde{y}_{3}$ is equal to zero. From the equilibrium theorem [1, p. 19] follows that in $(1.16)$ equality holds at least in two of the inequalities. In $\left(\widetilde{1.16^{\prime}}\right)$ at least one strict inequality holds. For if three equalities hold then by solving the system of equations we get $\widetilde{y}_{2}<0$, and so the solution is not feasible. Using again the equilibrium theorem we obtain that at least one of the numbers is equal to zero. As (1.14) holds, it follows that precisely one of those numbers is equal to zero. Summing up: In $(\widetilde{1.16})$ the sign of equality holds at least twice and precisely one of the numbers $\widetilde{x}_{1}, \widetilde{x}_{2}, \widetilde{x}_{3}$ vanishes.

We now consider all the sets $x_{1}, x_{2}, x_{3}$ for which the just obtained conditions hold. For every such set we decide whether it is a feasible solution (f.s) or whether it is not a feasible solution (n.f.s). For this decision we have to distinguish between the two following cases

$$
\begin{aligned}
& s_{1}+s_{2} \leqq s_{3}, \\
& s_{1}+s_{2} \geqq s_{3} .
\end{aligned}
$$

The result is given in the following table.

\begin{tabular}{c|c|c|c|c|c}
\hline $\begin{array}{c}\text { equality in (1.16) } \\
\text { in the equations }\end{array}$ & $x_{1}$ & $x_{2}$ & $x_{3}$ & $\begin{array}{c}\text { case } \\
(1.17)\end{array}$ & $\begin{array}{c}\text { case } \\
(1.18)\end{array}$ \\
\hline \hline$(1),(2)$ & 0 & $s_{1}-s_{2}$ & $s_{2}$ & n.f.s & n.f.s \\
\hline$(1),(2)$ & $s_{2}-s_{1}$ & 0 & $s_{1}$ & f.s & f.s \\
\hline$(1),(2)$ & $s_{2}$ & $s_{1}$ & 0 & f.s & n.f.s \\
\hline$(1),(3)$ & 0 & $s_{3}$ & $s_{1}-s_{3}$ & n.f.s & n.f.s \\
\hline$(1),(3)$ & $s_{3}$ & 0 & $s_{1}$ & n.f.s & n.f.s \\
\hline$(1),(3)$ & $s_{3}-s_{1}$ & $s_{1}$ & 0 & n.f.s & f.s \\
\hline$(2),(3)$ & 0 & $s_{3}$ & $s_{2}$ & n.f.s & n.f.s \\
\hline$(2),(3)$ & $s_{3}$ & 0 & $s_{2}-s_{3}$ & n.f.s & n.f.s \\
\hline$(2),(3)$ & $s_{2}$ & $s_{3}-s_{2}$ & 0 & n.f.s & f.s \\
\hline
\end{tabular}

For any row of this table containing a f.s, the limit case $s_{1}+s_{2}=s_{3}$ is to be associated with this f.s.

When (1.17) holds, the optimal solution is one of the following feasible solutions

$$
\begin{aligned}
& \left(x_{1}, x_{2}, x_{3}\right)=\left(s_{2}-s_{1}, 0, s_{1}\right), \\
& \left(x_{1}, x_{2}, x_{3}\right)=\left(s_{2}, s_{1}, 0\right) .
\end{aligned}
$$


As

$$
\begin{aligned}
M\left(s_{2}-s_{1}, 0, s_{1}\right) & =\left(s_{2}-s_{1}\right)\left(s_{2}-s_{3}\right)^{2}+s_{1}\left(s_{1}-s_{2}\right)^{2} \\
& <s_{2}\left(s_{2}-s_{3}\right)^{2}+s_{1}\left(s_{1}-s_{3}\right)^{2}=M\left(s_{2}, s_{1}, 0\right)
\end{aligned}
$$

it follows that

$$
\left(\widetilde{x}_{1}, \widetilde{x}_{2}, \widetilde{x}_{3}\right)=\left(s_{2}, s_{1}, 0\right)
$$

This optimal solution is unique.

When (1.18) holds, the optimal solution is one of the following feasible solutions

$$
\begin{aligned}
& \left(x_{1}, x_{2}, x_{3}\right)=\left(s_{2}-s_{1}, 0, s_{1}\right), \\
& \left(x_{1}, x_{2}, x_{3}\right)=\left(s_{3}-s_{1}, s_{1}, 0\right), \\
& \left(x_{1}, x_{2}, x_{3}\right)=\left(s_{2}, s_{3}-s_{2}, 0\right) .
\end{aligned}
$$

As

$$
\begin{gathered}
M\left(s_{3}-s_{1}, s_{1}, 0\right)-M\left(s_{2}, s_{3}-s_{2}, 0\right)=\left(s_{3}-s_{1}-s_{2}\right)\left(s_{2}-s_{3}\right)^{2} \\
+\left(s_{1}+s_{2}-s_{3}\right)\left(s_{1}-s_{3}\right)^{2} \geqq 0
\end{gathered}
$$

and

$$
\begin{aligned}
& M\left(s_{3}-s_{1}, s_{1}, 0\right)=\left(s_{3}-s_{1}\right)\left(s_{2}-s_{3}\right)^{2}+s_{1}\left(s_{1}-s_{3}\right)^{2} \\
& \quad>\left(s_{2}-s_{1}\right)\left(s_{2}-s_{3}\right)^{2}+s_{1}\left(s_{2}-s_{1}\right)^{2}=M\left(s_{2}-s_{1}, 0, s_{1}\right),
\end{aligned}
$$

it follows that

$$
\left(\widetilde{x}_{1}, \tilde{x}_{2}, \widetilde{x}_{3}\right)=\left(s_{3}-s_{1}, s_{1}, 0\right) .
$$

As equality in (1.20) holds only if $s_{1}+s_{2}=s_{3}$, it follows that the optimal solution (1.21) is unique. We remark that the optimal solution can also be determined by the simplex method [1, ch. 4]. According to (1.9), (1.19) and (1.21) we have to prove that

$$
M\left(s_{2}, s_{1}, 0\right)=s_{2}\left(s_{2}-s_{3}\right)^{2}+s_{1}\left(s_{1}-s_{3}\right)^{2}<\frac{1}{3} \sum_{1 \leqq i<j}^{3}\left(s_{i}+s_{j}\right)\left(s_{i}-s_{j}\right)^{2}
$$

when (1.17) holds, and that

$$
\begin{aligned}
& M\left(s_{3}-s_{1}, s_{1}, 0\right) \\
& \quad=\left(s_{3}-s_{1}\right)\left(s_{2}-s_{3}\right)^{2}+s_{1}\left(s_{1}-s_{3}\right)^{2}<\frac{1}{3} \sum_{1 \leqq i<j}^{3}\left(s_{i}+s_{j}\right)\left(s_{i}-s_{j}\right)^{2}
\end{aligned}
$$

when (1.18) holds.

Denote

$$
s_{1}=\alpha, s_{2}=\alpha+\beta, s_{3}=\alpha+\beta+\gamma \text {. }
$$


The assumption (1.14) implies

$$
\alpha, \beta, \gamma>0 \text {. }
$$

Assuming the validity of (1.17), we prove now that (1.22) holds. (1.17) gives

$$
\alpha \leqq \gamma
$$

Denote

$$
\begin{aligned}
I_{1} & =\sum_{1 \leq i<j}^{3}\left(s_{i}+s_{j}\right)\left(s_{i}-s_{j}\right)^{2}-3 M\left(s_{2}, s_{1}, 0\right) \\
& =\left(s_{1}-s_{2}\right)^{2}\left(s_{1}+s_{2}\right)+\left(s_{2}-s_{3}\right)^{2}\left(s_{3}-2 s_{2}\right)+\left(s_{3}-s_{1}\right)^{2}\left(s_{3}-2 s_{1}\right) .
\end{aligned}
$$

By the notation of (1.24) $I_{1}$ takes the form

(1.27) $I_{1}=\beta^{2}(2 \alpha+\beta)+\gamma^{2}(\gamma-\alpha-\beta)+(\beta+\gamma)^{2}(\beta+\gamma-\alpha)$.

From (1.25), (1.26) and (1.27) follows

$$
I_{1}>\beta^{2}(2 \alpha+\beta)+\gamma^{2}(2 \gamma-2 \alpha)>0 .
$$

(1.22) is thus established.

Assuming the validity of (1.18), we prove that (1.23) holds. (1.18) gives

$$
\alpha \geqq \gamma
$$

Denote

$$
\begin{aligned}
I_{2} & =\sum_{1 \leq i<j}^{3}\left(s_{i}+s_{j}\right)\left(s_{i}-s_{j}\right)^{2}-3 M\left(s_{3}-s_{1}, s_{1}, 0\right) \\
& =\left(s_{1}-s_{2}\right)^{2}\left(s_{1}+s_{2}\right)+\left(s_{2}-s_{3}\right)^{2}\left(3 s_{1}+s_{2}-2 s_{3}\right)+\left(s_{1}-s_{3}\right)^{2}\left(s_{3}-2 s_{1}\right) .
\end{aligned}
$$

By the notation of (1.24) $I_{2}$ takes the form

(1.29) $I_{2}(\alpha, \beta, \gamma)=\beta^{2}(2 \alpha+\beta)+\gamma^{2}(2 \alpha-\beta-2 \gamma)+(\beta+\gamma)^{2}(\beta+\gamma-\alpha)$.

We distinguish between the following two cases

$$
\begin{aligned}
& \beta+\gamma \geqq \alpha, \\
& \beta+\gamma<\alpha .
\end{aligned}
$$

At first assume that (1.30) holds. From (1.25), (1.28), (1.29) and (1.30) we obtain

$$
\begin{array}{r}
I_{2} \geqq \beta^{2}(2 \alpha+\beta)+\gamma^{2}(2 \alpha-\beta-2 \gamma)+\gamma^{2}(\beta+\gamma-\alpha) \\
=\beta^{2}(2 \alpha+\beta)+\gamma^{2}(\alpha-\gamma)>0 .
\end{array}
$$

(1.23) is thus established when (1.30) holds. Assume now that (1.31) 
holds. Write $I_{2}(\alpha, \beta, \gamma)$ in the following form

$$
I_{2}(\alpha, \beta, \gamma)=\alpha(\beta-\gamma)^{2}+\beta^{3}+(\beta+\gamma)^{3}-\gamma^{2}(\beta+2 \gamma) .
$$

$I_{\Sigma}(\alpha, \beta, \gamma)$ is linear in $\alpha$. Let $\beta, \gamma$ be any constant positive numbers. As

$$
I_{2}(\alpha, \gamma, \gamma)=6 \gamma^{3}>0
$$

we may assume that

$$
(\beta-\gamma)^{2}>0
$$

Using the validity of (1.23) when (1.30) holds, we obtain

$$
I_{2}(\beta+\gamma, \beta, \gamma)>0 \text {. }
$$

From (1.32) and (1.33) it follows that

$$
\lim _{\alpha \rightarrow+\infty} I_{2}(\alpha, \beta, \gamma)=+\infty \text {. }
$$

As $I_{2}(\alpha, \beta, \gamma)$ is linear in $\alpha$, it follows from (1.34) and (1.35) that $I_{2}(\alpha, \beta, \gamma)>0$ when (1.31) holds. (1.23) is thus established also when (1.31) holds.

The proof of the theorem is completed in the case when (1.14) holds. We proved that in this case (1.1) holds strictly. From continuity considerations it follows that the theorem without the equality statement holds also if only (1.13) is assumed. (We have already mentioned that (1.13) can be considered as the general case). Hence, to complete our proof in the general case (1.13), we have to assume that (1.14) is invalidated and to check for possible cases of equality in (1.1). If (1.14) does not hold, there are three possibilities:

$$
\begin{aligned}
& \text { (1) } s_{1}=s_{2}=s_{3}, \\
& \text { (2) } s_{1}<s_{2}=s_{3}, \\
& \text { (3) } s_{1}=s_{2}<s_{3} .
\end{aligned}
$$

If (1) holds then the sign of equality in (1.1) holds for every $A$. In this case $A$ is a generalized stochastic matrix.

In cases (2) and (3) we consider the corresponding maximum problems.

The maximum problem corresponding to (2) is: Maximize

$$
M\left(x_{1}, x_{2}, x_{3}\right)=\left(s_{1}-s_{3}\right)^{2}\left(x_{2}+x_{3}\right),
$$

where $x_{i} \geqq 0, i=1,2,3$, satisfy the three inequalities

$$
\left\{\begin{array}{l}
x_{2}+x_{3} \leqq s_{1} \\
x_{1}+x_{3} \leqq s_{3} \\
x_{1}+x_{2} \leqq \\
s_{3}
\end{array} .\right.
$$


It is obvious that every feasible solution for which $x_{2}+x_{3}=s_{1}$ is an optimal solution. So there are infinitely many optimal solutions. If in this case the sign of equality holds in (1.1), then

$$
s_{1}\left(s_{1}-s_{3}\right)^{2}=\frac{2}{3}\left(s_{1}-s_{3}\right)^{2}\left(s_{1}+s_{3}\right),
$$

and therefore

$$
s_{1}=2 s_{3} \text {. }
$$

As the last equality contradicts (1.13), we conclude that in the case

(2) strict inequality holds in (1.1).

The maximum problem corresponding to (3) is: Maximize

$$
M\left(x_{1}, x_{2}, x_{3}\right)=\left(x_{1}+x_{2}\right)\left(s_{1}-s_{3}\right)^{2},
$$

where $x_{i} \geqq 0, i=1,2,3$, satisfy the three inequalities

$$
\left\{\begin{array}{l}
x_{2}+x_{3} \leqq s_{1} \\
x_{1}+x_{3} \leqq s_{1} \\
x_{1}+x_{2} \leqq s_{3}
\end{array}\right.
$$

In order to determine optimal solutions of the problem; we have to distinguish between the following two cases

$$
\begin{array}{ll}
(3)_{I} \quad 2 s_{1} \leqq s_{3}, \\
(3)_{I I} \quad 2 s_{1}>s_{3} .
\end{array}
$$

If $(3)_{I}$ holds then the only optimal solution is

$$
\left(\widetilde{x}_{1}, \widetilde{x}_{2}, \widetilde{x}_{3}\right)=\left(s_{1}, s_{1}, 0\right) \text {. }
$$

If $(3)_{I I}$ holds then every feasible solution for which $x_{1}+x_{2}=s_{3}$ is an optimal solution. In this case there are infinitely many optimal solutions. If the sign of equality in (1.1) holds in the case $(3)_{I}$ then

$$
2 s_{1}\left(s_{1}-s_{3}\right)^{2}=\frac{2}{3}\left(s_{1}+s_{3}\right)\left(s_{1}-s_{3}\right)^{2},
$$

and therefore

$$
2 s_{1}=s_{3} \text {. }
$$

If the sign of equality in (1.1) holds in the case $(3)_{I I}$ then

$$
s_{3}\left(s_{1}-s_{3}\right)^{2}=\frac{2}{3}\left(s_{1}+s_{3}\right)\left(s_{1}-s_{3}\right)^{2},
$$

and (1.36) is obtained again. As (1.36) contradicts (3) $)_{I I}$, it follows 
that in this case equality in (1.1) is excluded. Hence, in case (3) equality in (1.1) holds if and only if

$$
s_{1}=s_{2}, s_{3}=2 s_{1},\left(\widetilde{x}_{1}, \widetilde{x}_{2}, \widetilde{x}_{3}\right)=\left(s_{1}, s_{1}, 0\right),
$$

that is only for the matrix

$$
A=\left[\begin{array}{ccc}
0 & 0 & s_{1} \\
0 & 0 & s_{1} \\
s_{1} & s_{1} & 0
\end{array}\right] .
$$

$A^{2}$ is a generalized stochastic matrix (while $A$ is not stochastic). It follows from (1.3) that if $A$ or $A^{2}$ is a generalized stochastic matrix then equality in (1.1) holds. Hence, it follows that equality in (1.1) holds if and only if $A$ or $A^{2}$ is a generalized stochastic matrix. This completes the proof of the theorem.

REMARK 1. The following example proves that the assumption of symmetry in Theorem 1 is essential. Let

$$
A=\left[\begin{array}{lll}
1 & 2 & 1 \\
1 & 1 & 2 \\
0 & 1 & 1
\end{array}\right] .
$$

$A$ is a positive nonsymmetric matrix. As

$$
S(A)=10, S\left(A^{2}\right)=32, S\left(A^{3}\right)=100,
$$

(1.1) does not hold.

It is obvious that (1.1) does not hold in general for real symmetric matrices with (some) negative elements. However, going over to the absolute values and denoting $|A|=\left(\left|a_{i j}\right|\right)$, one may think that for all $n \times n, n \leqq 3$, symmetric matrices

$$
S(|A|) S\left(\left|A^{2}\right|\right) \leqq n S\left(\left|A^{3}\right|\right)
$$

holds. The following counter example shows that this is wrong. Let

$$
A=\left[\begin{array}{rrr}
1 & -2 & 1 \\
-2 & 0 & 2 \\
1 & 2 & -1
\end{array}\right]
$$

As

$$
S(|A|)=12, \quad S\left(\left|A^{2}\right|\right)=36, \quad S\left(\left|A^{3}\right|\right)=128,
$$

$\left(1.1^{\prime}\right)$ does not hold. 
REMARK 2. Let $A$ be a $3 \times 3$ nonnegative symmetric matrix. Let $r_{1}, r_{2}, r_{3}$ be an orthonormal system of characteristic vectors of $A$ corresponding respectively to the characteristic values $\alpha_{1}, \alpha_{2}, \alpha_{3}$. Let $R$ be the orthogonal matrix with the columns $r_{1}, r_{2}, r_{3}$. As $A=R D R^{r}$, where $D$ is the diagonal matrix $\left\{\alpha_{1}, \alpha_{2}, \alpha_{3}\right\}$ and $R^{T}$ is the transposed of $R$, we have

$$
S\left(A^{m}\right)=\left(A^{m} e, e\right)=\left(D^{m} R^{T} e, R^{T} e\right)=\sum_{i=1}^{3} \alpha_{i}^{m}\left[S\left(r_{i}\right)\right]^{2} .
$$

Hence, (1.1) for $n=3$ is transformed to

$$
\sum_{i=1}^{3} \alpha_{i}\left[S\left(r_{i}\right)\right]^{2} \sum_{i=1}^{3} \alpha_{i}^{2}\left[S\left(r_{i}\right)\right]^{2} \leqq 3 \sum_{i=1}^{3} \alpha_{i}^{3}\left[S\left(r_{i}\right)\right]^{2} .
$$

(1.38) is a necessary condition for a system of 3 orthonormal vectors $r_{1}, r_{2}, r_{3}$ and three real numbers $\alpha_{1}, \alpha_{2}, \alpha_{3}$ to be respectively a system of characteristic vectors and values of a $3 \times 3$ nonnegative symmetric matrix. It would be interesting to find similar necessary (or sufficient) conditions concerning $n \times n$ nonnegative symmetric matrices.

REMARK 3. From the considerations concerning the equality sign in the proof of Theorem 1 we conclude: Let $A$ be $a 3 \times 3$ nonnegative symmetric matrix satisfying (1.13). $A$ is not generalized stochastic while $A^{2}$ is generalized stochastic if and only if $A$ is of the form (1.37). In a recent paper [3] we characterize the matrices of this type for every $n$.

1.3. Counter example for $n \geqq 4$. In this section we bring a counter example which shows that for $n \geqq 4$ the conjecture of Marcus and Newman does not hold. Let

$$
A_{n}=A_{n}(\alpha)=\left[\begin{array}{ccccc}
\alpha & 0 & - & - & 0 \\
0 & 0 & & 0 & 1 \\
1 & 1 & 1 & 1 & 1 \\
1 & 1 & 1 & 1 & 1 \\
1 & 1 & 1 & 1 & 1 \\
1 & 1 & 1 & 1 & 1 \\
1 & 0 & - & 0 & 1 \\
0 & 1 & - & 1 & 0
\end{array}\right]=\left[\begin{array}{cccc}
\alpha & 0 & -\cdots-\cdots 0 \\
0 & \\
1 & \\
1 & B_{n-1} \\
1 & \\
1 & \\
1 & \\
0 &
\end{array}\right], n \geqq 4
$$

$A_{n}(\alpha)$ is a $n \times n$ symmetric matrix depending on the real parameter $\alpha$. For $\alpha \geqq 0 A_{n}(\alpha)$ is nonnegative. $B_{n-1}$ is a $(n-1) \times(n-1)$ nonnegative symmetric matrix. $B_{n-1}^{2}$ is generalized stochastic (while $B_{n-1}$ is not generalized stochastic). As 


$$
\begin{array}{ll}
s\left(B_{n-1}^{2}\right)=(n-2) e, \quad & S\left(B_{n-1}^{2}\right)=(n-1)(n-2), \\
S\left(B_{n-1}\right)=2(n-2), & S\left(B_{n-1}^{3}\right)=2(n-2)^{2},
\end{array}
$$

we obtain

$$
\begin{aligned}
n S( & \left.A_{n}^{3}\right)-S\left(A_{n}\right) S\left(A_{n}^{2}\right)=n\left[2(n-2)^{2}+\alpha^{3}\right] \\
& \quad-[2(n-2)+\alpha]\left[(n-1)(n-2)+\alpha^{2}\right] \\
= & {\left[\alpha^{2}-(n-2)\right][(n-1) \alpha-2(n-2)]=f_{n}(\alpha) . }
\end{aligned}
$$

The zeros of the polynomial $f_{n}(\alpha)$ are

$$
\alpha_{1}=-\sqrt{n-2}, \quad \alpha_{2}=\frac{2(n-2)}{n-1}, \quad \alpha_{3}=+\sqrt{n-2},
$$

and therefore $f_{n}(\alpha)<0$ for

$$
\frac{2(n-2)}{n-1}<\alpha<\sqrt{n-2} \text {. }
$$

Hence, for every $\alpha$ satisfying (1.40) the inequality (1.1) does not hold.

REMARK. Consider the following generalization of conjecture (1.1): Let $A$ be a $n \times n$ nonnegative symmetric matrix. Then

$$
S(A) S\left(A^{m}\right) \leqq n S\left(A^{m+1}\right), \quad m=1,2, \cdots .
$$

For odd $m$ (1.41) holds for every symmetric $A$ [4, Th. 4]. For even $m$ and $n \geqq 4$ a straightforward computation proves that (1.41) does not hold for the matrices (1.39), for $\alpha$ satisfying (1.40). For $m=2$ and $n \leqq 3$ the validity of (1.41) is established in Theorem 1. For even $m>2$ and $n=3$ the problem remains open.

2. Upper bound for the sum of the elements of a power of a matrix.

2.1. A conjecture. In this section we state a conjecture which yields an upper bound for the sum of the elements of a power of a nonnegative symmetric matrix.

We first define a class of matrices: Let $s=\left(s_{1}, \cdots, s_{n}\right)$ be a vector for which the condition

$$
0<s_{1}<s_{2}<\cdots<s_{n}
$$

holds. Denote by $\mathscr{A}_{n}(s)$ the class of all $n \times n$ nonnegative symmetric matrices for which $s(A)=s$.

By a straightforward computation, using (1.2), (1.4) and (1.5), we obtain 


$$
S\left(A^{3}\right)=\sum_{i=1}^{n} s_{i}^{3}-\sum_{1 \leq i<j}^{n} a_{i j}\left(s_{i}-s_{j}\right)^{2}
$$

From (2.2) it follows that for every $A \in \mathscr{A}_{n}(s)$ the inequality

$$
S\left(A^{3}\right) \leqq \sum_{i=1}^{n} s_{i}^{3}
$$

holds. Equality in (2.3) holds if and only if $A$ is the diagonal matrix in $\mathscr{A}_{n}(s)$.

The following conjecture generalizes (2.3): For every $A \in \mathscr{A}_{n}(s)$ the inequality

$$
S\left(A^{m}\right) \leqq \sum_{i=1}^{n} s_{i}^{m}, \quad m=3,4, \cdots
$$

holds. Equality in (2.4) holds if and only if $A$ is the diagonal matrix in $\mathscr{A}_{n}(s)$.

REMARK 1. For $m=1,2(2.4)$ holds with equality sign for every $A \in \mathscr{A}_{n}(s)$. This is the reason why we did not include $m=1,2$ in our formulation of the conjecture.

REMARK 2. In the definition of the class $\mathscr{A}_{n}(s)$ we assumed that $s(A)$ satisfies (2.1). If we ommit this assumption only the equality statement of the conjecture is to be changed.

2.2. Proof for particular cases. In this section we prove some particular cases of the conjecture. The general case remains open.

THEOREM 2. In the following two cases

(1) $m=3,4,5$ and $n=1,2, \cdots$

(2) $m=3,4, \cdots$ and $n=1,2,3$

the inequality

$$
S\left(A^{m}\right) \leqq \sum_{i=1}^{n} s_{i}^{m}
$$

holds for every $A \in \mathscr{A}_{n}(s)$. The equality sign in these two cases holds only for the diagonal matrix in $\mathscr{A}_{n}(s)$.

Proof. Let $A=\left(a_{i j}\right) \in \mathscr{A}_{n}(s)$. Assume that there exists an $i$, $1 \leqq i<n$, for which $a_{n i}>0$. Define 


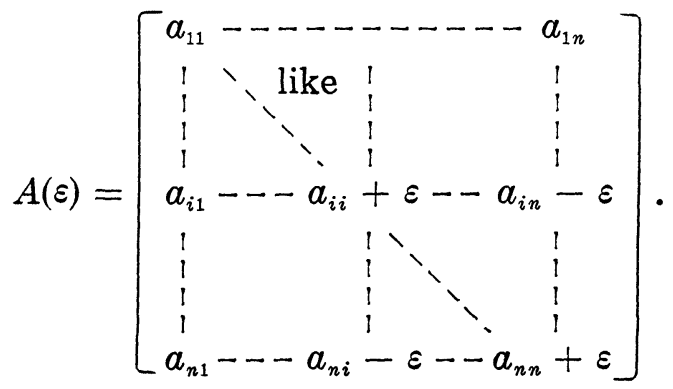

Here $\varepsilon$ is a nonnegative parameter. For small $\varepsilon A(\varepsilon) \in \mathscr{A}_{n}(s)$. We have

$$
\begin{gathered}
\left.\frac{d S\left[A^{m}(\varepsilon)\right]}{d \varepsilon}\right|_{\varepsilon=0}=S\left[\frac{d A^{m}(\varepsilon)}{d \varepsilon}\right]_{\varepsilon=0}=\sum_{k=0}^{m-1} S\left[A^{k} A^{\prime}(0) A^{m-k-1}\right] \\
=\sum_{k=0}^{m-1}\left(A^{\prime}(0) A^{m-k-1} e, A^{k} e\right)=\sum_{k=0}^{m-1}\left(A^{\prime}(0) s^{(m-k-1)}(A), s^{(k)}(A)\right) \\
=\sum_{k=0}^{m-1}\left[s_{i}^{(m-k-1)}(A)-s_{n}^{(m-k-1)}(A)\right]\left[s_{i}^{(k)}(A)-s_{n}^{(k)}(A)\right] .
\end{gathered}
$$

Hence,

(2.6) $\left.\frac{d S\left[A^{m}(\varepsilon)\right]}{d \varepsilon}\right|_{\varepsilon=0}=\sum_{k=1}^{m-2}\left[s_{i}^{(m-k-1)}(A)-s_{n}^{(m-k-1)}(\dot{A})\right]\left[s_{i}^{(k)}(A)-s_{n}^{(k)}(A)\right]$.

Let us first bring the proof for the case (1). Let $\tilde{A}=\widetilde{A}(m)=\left(\widetilde{a}_{i j}\right)$, $m=3,4,5$, be an optimal matrix of the maximum problem

$$
\operatorname{Max}_{A \in \mathscr{A}_{n}^{(s)}} S\left(A^{m}\right)
$$

For a fixed $m, m=3,4,5$, we use induction on $n$. For $n=1$ the theorem holds trivially. Suppose that the theorem holds for $n-1$ (and the same fixed $m$ ). We prove shortly that the optimal $n \times n$ matrix $\widetilde{A}$ has the following structure

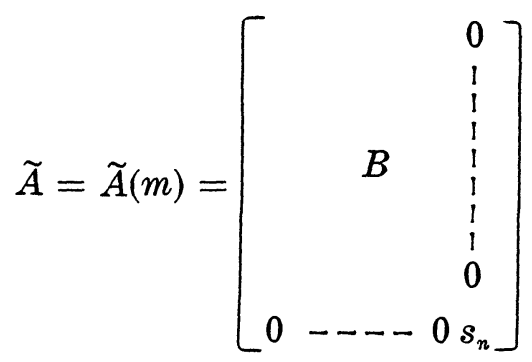

$B$ is a $(n-1) \times(n-1)$ nonnegative symmetric matrix and $s(B)=$ $\left(s_{1}, \cdots, s_{n-1}\right)$. Suppose that we have already proved that $\widetilde{A}$ has the structure (2.7). By the induction assumption

$$
S\left(B^{m}\right) \leqq \sum_{i=1}^{n-1} s_{i}^{m}
$$


and equality holds only if $B$ is diagonal. Hence,

$$
S\left(\widetilde{A}^{m}\right)=S\left(B^{m}\right)+s_{n}^{m} \leqq \sum_{i=1}^{n} s_{i}^{m} .
$$

Equality in (2.8) holds if and only if $\tilde{A}$ is a diagonal matrix.

It remains to prove that $\tilde{A}$ has the structure (2.7). Assume that $\tilde{A}$ has not the above structure. There exists at least one $i, 1 \leqq i \leqq n-1$, for which $\tilde{a}_{n i}>0$. For this $i$ the matrix $\widetilde{A}(\varepsilon)$ is defined according to (2.5). As $\widetilde{A}$ is an optimal matrix of the above defined maximum problem, and as for a small enough $\varepsilon>0 \widetilde{A}(\varepsilon) \in \mathscr{A}_{n}(s)$, the inequality

$$
\left.\frac{S d\left[\widetilde{A}^{m}(\varepsilon)\right]}{d \varepsilon}\right|_{\varepsilon=0} \leqq 0
$$

must hold. From (2.6) and (2.9) we obtain

$$
\sum_{k=1}^{m-2}\left[s_{i}^{(k)}(\tilde{A})-s_{n}^{(k)}(\widetilde{A})\right]\left[s_{i}^{(m-k-1)}(\widetilde{A})-s_{n}^{(m-k-1)}(\widetilde{A})\right] \leqq 0 .
$$

We now consider separately the cases $m=3,4,5$. By a suitable choice of $i$ we obtain a contradiction to (2.10).

$m=3$. For this case the theorem has already been proved by the representation (2.2). We give here an independent proof. Choose any $i, 1 \leqq i \leqq n-1$, for which $\widetilde{a}_{n i}>0$. According to our assumption there exists such an $i$. By (2.10) we obtain for this $i$

$$
\left[s_{i}(\tilde{A})-s_{n}(\tilde{A})\right]^{2}=\left(s_{i}-s_{n}\right)^{2} \leqq 0 .
$$

(2.11) contradicts (2.1).

$m=4$. Let $i, 1 \leqq i \leqq n-1$, be the smallest index for which $\tilde{a}_{n i}>0$. According to our assumption there exists such an $i$. By (2.10) we obtain for this $i$

$$
\left(s_{n}-s_{i}\right)\left[s_{n}^{(2)}(\tilde{A})-s_{i}^{(2)}(\tilde{A})\right] \leqq 0 .
$$

We have

$$
\widetilde{A} s=s^{(2)}(\widetilde{A}) .
$$

By (2.1) and by our choice of $i$ we obtain

$$
\begin{aligned}
& s_{i}^{(2)}(\widetilde{A})=\sum_{j=1}^{n} \widetilde{a}_{i j} s_{j} \leqq s_{i} s_{n}, \\
& s_{n}^{(2)}(\widetilde{A})=\sum_{j=1}^{n} \widetilde{a}_{n j} s_{j} \geqq s_{i} s_{n} .
\end{aligned}
$$

Hence, 


$$
s_{i}^{(2)}(\widetilde{A}) \leqq s_{n}^{(2)}(\widetilde{A})
$$

Equality in (2.15) implies equality in (2.13) and (2.14). Equality in (2.13) holds if and only if

$$
\tilde{a}_{i 1}=\cdots=\tilde{a}_{i, n-1}=0, \tilde{a}_{i n}=s_{i} .
$$

Equality in (2.14) holds if and only if

$$
\tilde{a}_{n, i+1}=\cdots=\tilde{a}_{n n}=0, \tilde{a}_{n i}=s_{n} .
$$

Hence,

$$
\tilde{a}_{i n}=\tilde{a}_{n i}=s_{i}=s_{n} .
$$

(2.16) contradicts (2.1) and therefore (2.15) holds strictly. (2.1) and the strict inequality in (2.15) contradict (2.12).

$m=5$. From the set of all the indices $i, 1 \leqq i \leqq n$, for which $\tilde{a}_{n i}>0$ choose that $i$ for which $s_{i}^{(2)}(\widetilde{A})$ attains its minimum value. According to our assumption there exists an $i, 1 \leqq i<n$, for which $\tilde{a}_{n i}>0$. As we saw in the proof for $m=4$, there exists an $i, 1 \leqq$ $i<n$, which satisfies $\tilde{a}_{n i}>0$ and for which strict inequality holds in (2.15). It follows that the $i$ chosen now satisfies $i<n$. By (2.10) we obtain for this $i$

$$
\dot{2}\left(s_{n}-s_{i}\right)\left[s_{n}^{(3)}(\tilde{A})-s_{i}^{(3)}(\tilde{A})\right]+\left[s_{n}^{(2)}(\tilde{A})-s_{i}^{(2)}(\tilde{A})\right]^{2} \leqq 0 .
$$

We have

$$
s^{(3)}(\widetilde{A})=\widetilde{A}^{3} e=\widetilde{A} s^{(2)}(\widetilde{A})=\widetilde{A}^{2} s(\widetilde{A}) .
$$

By (2.1) and by our choice of $i$ we obtain

$$
\begin{aligned}
& s_{n}^{(3)}(\widetilde{A})=\sum_{j=1}^{n} \widetilde{a}_{n j} s_{j}^{(2)} \geqq s_{i}^{(2)}(\widetilde{A}) s_{n}, \\
& s_{i}^{(3)}(\widetilde{A})=\sum_{j=1}^{n} \widetilde{a}_{i j}^{(2)} s_{j}<s_{i}^{(2)}(\widetilde{A}) s_{n} .
\end{aligned}
$$

As $\widetilde{a}_{n i} \neq 0$, it follows that $\widetilde{a}_{i i}^{(2)} \neq 0$. As $\widetilde{a}_{i i}^{(2)} \neq 0$ and as $i<n$, it follows that the strict inequality sign in (2.19) is justified. (2.18) and (2.19) imply

$$
s_{i}^{(3)}(\widetilde{A})<s_{n}^{(3)}(\widetilde{A}) \text {. }
$$

(2.1) and (2.20) contradict (2.17). The proof of the case (1) is thus completed.

We bring now the proof for the case (2). We give first the proof for $n=3$. Let $\widetilde{A}=\widetilde{A}(m), m=3,4, \cdots$, be an optimal matrix of the problem 


$$
\underset{A \in \mathscr{A}_{3}(s)}{\operatorname{Max}} S\left(A^{m}\right)
$$

Assume that $\tilde{A}(m)$, for a fixed $m$ from $m=3,4, \cdots$, has not the structure (2.7). There are then two possibilities:

$$
\begin{aligned}
& \tilde{a}_{31} \neq 0, \\
& \tilde{a}_{31}=0, \widetilde{a}_{32} \neq 0 .
\end{aligned}
$$

If (2.21) holds then, according to (2.10), it is sufficient to prove that for every natural $k$ the inequality

$$
s_{1}^{(k)}(\widetilde{A})<s_{3}^{(k)}(\widetilde{A})
$$

holds, while if $(2.22)$ holds it is sufficient to prove that

$$
s_{2}^{(k)}(\tilde{A})<s_{3}^{(k)}(\widetilde{A}) \text {. }
$$

Assume that (2.21) holds. As

(2.25) $\quad s_{i}^{(k)}(\widetilde{A})=\sum_{j=1}^{3} \widetilde{a}_{i j} s_{j}^{(k-1)}(\widetilde{A})=\sum_{j=1}^{3} \widetilde{a}_{i j}^{(k-1)} s_{j}, i=1,2,3 ; k=2,3, \cdots$, it follows that

$$
\begin{aligned}
& s_{1}^{(k)}(\widetilde{A}) \leqq \min \left\{s_{3} s_{1}^{(k-1)}(\widetilde{A}), s_{1} \max _{j} s_{j}^{(k-1)}(\widetilde{A})\right\}, \\
& s_{3}^{(k)}(\widetilde{A}) \geqq \max \left\{s_{1} s_{3}^{(k-1)}(\widetilde{A}), s_{3} \min _{j} s_{j}^{(k-1)}(\widetilde{A})\right\} .
\end{aligned}
$$

We prove (2.23) by induction on $k$. For $k=1(2.23)$ holds by (2.1). Assume that

$$
s_{1}^{(k-1)}(\widetilde{A})<s_{3}^{(k-1)}(\widetilde{A}) .
$$

From this induction assumption follows that at least one of the two following equations holds:

$$
\begin{aligned}
& s_{1}^{(k-1)}(\widetilde{A})=\min _{j} s_{j}^{(k-1)}(\widetilde{A}), \\
& s_{3}^{(k-1)}(\widetilde{A})=\max _{j} s_{j}^{(k-1)}(\widetilde{A}) .
\end{aligned}
$$

The minimum and the maximum are strict. As (2.28) or (2.29) holds, it follows from (2.26) and (2.27) that

$$
s_{1}^{(k)}(\tilde{A}) \leqq s_{3}^{(k)}(\widetilde{A}) \text {. }
$$

To obtain (2.23) we have to show that equality cannot hold in $\left(2.23^{\prime}\right)$. Assume that (2.28) holds. Equality in (2.23') implies

$$
s_{3}^{(k)}(\tilde{\widetilde{A}})=s_{3} s_{1}^{(k-1)}(\tilde{A}) \text {. }
$$


From the last equation, using (2.25) and the fact that the minimum in (2.28) is strict, we obtain

$$
\tilde{a}_{32}=\tilde{a}_{33}=0, \widetilde{a}_{31}=s_{3}=\tilde{a}_{13} \leqq s_{1} .
$$

(2.30) contradicts (2.1). Assume that (2.29) holds. Similar to our last conclusion it follows now that equality in (2.23') implies

$$
\tilde{a}_{11}=\tilde{a}_{12}=0, \tilde{a}_{13}=s_{1}, \quad \tilde{a}_{32}^{(k-1)}=\tilde{a}_{33}^{(k-1)}=0 .
$$

As from $\widetilde{a}_{33} \neq 0$ follows $\widetilde{a}_{33}^{(k-1)} \neq 0$, we obtain

$$
\tilde{a}_{33}=0 \text {. }
$$

If $\widetilde{a}_{32} \neq 0$, using $(2.31)$ and $(2.32)$, we obtain

$$
\begin{cases}\widetilde{a}_{33}^{(k-1)} \neq 0, & k-1 \text { even } \\ \tilde{a}_{32}^{(k-1)} \neq 0, & k-1 \text { odd }\end{cases}
$$

(2.33) follows easily, e.g. from the directed graph corresponding to $\tilde{A}$. (2.33) contradicts $(2.31)$ and therefore $\tilde{a}_{32}=0$. We obtained

$$
\widetilde{a}_{32}=\widetilde{a}_{33}=0, \tilde{a}_{13}=\widetilde{a}_{31}=s_{1}=s_{3} \text {. }
$$

(2.34) contradicts (2.1). So (2.23) holds and the proof for this case is completed.

Assume that (2.22) holds. We prove $(2.24)$ by induction on $k$. Assume that

$$
\boldsymbol{s}_{2}^{(k-1)}(\widetilde{A})<\boldsymbol{s}_{3}^{(k-1)}(\tilde{A}) .
$$

From (2.22), (2.25) and (2.35) follows

$$
\begin{aligned}
& s_{2}^{(k)}(\widetilde{A}) \leqq s_{3} s_{2}^{(k-1)}(\widetilde{A}), \\
& s_{3}^{(k)}(\widetilde{A}) \geqq s_{3} s_{2}^{(k-1)}(\widetilde{A}) .
\end{aligned}
$$

Hence,

$$
s_{2}^{(k)}(\widetilde{A}) \leqq s_{3}^{(k)}(\widetilde{A}) \text {. }
$$

To obtain (2.24) we have to show that equality cannot hold in $\left(2.24^{\prime}\right)$. Equality in $\left(2.24^{\prime}\right)$ implies equality in (2.36) and this implies $\tilde{a}_{33}=0$. So we have

$$
\tilde{a}_{31}=\widetilde{a}_{33}=0, \tilde{a}_{32}=s_{3}=\tilde{a}_{23} \leqq s_{2} .
$$

(2.37) contradicts (2.1). So (2.24) holds and the proof for $n=3$ is completed.

For $n=2$ it is sufficient to prove that for every natural $k$

$$
s_{1}^{(k)}(\tilde{A})<s_{2}^{(k)}(\tilde{A}) \text {. }
$$


This inequality can be easily proved by induction. Theorem 2 is thus established.

REMARK. It is easy to prove that if $A$ is a nonnegative matrix with row sums $s_{1}, \cdots, s_{n} ; s_{1} \leqq s_{2} \leqq \cdots \leqq s_{n}$, then

$$
s_{1}^{m-1} S(A) \leqq S\left(A^{m}\right) \leqq s_{n}^{m-1} S(A), \quad m=1,2, \cdots,
$$

where the two bounds are sharp. As for $A \in \mathscr{A}_{n}(s)$

$$
\sum_{i=1}^{n} s_{i}^{m}<s_{n}^{m-1} S(A)
$$

and as the bound $s_{n}^{m-1} S(A)$ is sharp, it follows that the assumption of symmetry in Theorem 2 is essential.

2.3. Generalizations. Theorem 2 can be generalized to a larger class of matrices and also to a statement on minors of matrices.

Let $A=\left(a_{i j}\right)$ be a $n \times n$ matrix, perhaps with complex elements. Denote $|A|=\left(\left|a_{i j}\right|\right)$. The row sums vector of $|A|, s(|A|)$, is denoted by $[s]=[s](A)$. The ith component of $[s]$ is denoted by $\left[s_{i}\right]=\left[s_{i}\right](A)$.

We bring now the first generalization of Theorem 2: In the following two cases

(1) $\quad m=3,4,5$ and $n=1,2, \cdots$

(2) $m=3,4, \cdots$ and $n=1,2,3$

the inequality

$$
S\left(\left|A^{m}\right|\right) \leqq \sum_{i=1}^{n}\left[s_{i}\right]^{m}
$$

holds for every complex $A$ such that $|A| \in \mathscr{A}_{n}([s])$. The equality sign in these two cases holds if and only if $A$ is diagonal.

Proof. We have

$$
\begin{aligned}
S\left(\left|A^{m}\right|\right) & =\sum_{i, j=1}^{n}\left|\sum_{k_{1}, \cdots, k_{m-1}=1}^{n} a_{i k_{1}} a_{k_{1} k_{2}} \cdots a_{k_{m-1}, j}\right| \\
& \leqq \sum_{i, j=1}^{n} \sum_{k_{1}, \cdots, k_{m-1}=1}^{n}\left|a_{i k_{1}} a_{k_{1} k_{2}} \cdots a_{k_{m-1}, j}\right|=S\left(|A|^{m}\right) .
\end{aligned}
$$

As $|A| \in \mathscr{A}_{n}([s])$ it follows from Theorem 2 that

$$
S\left(|A|^{m}\right) \leqq \sum_{i=1}^{n}\left[s_{i}\right]^{m}
$$

(2.39) and (2.40) imply (2.38). The equality statement follows from the equality statement in Theorem 2. 
Remark 1. For $A \in \mathscr{A}_{n}(s)$ (2.38) reduces to $\left(2.4^{\prime}\right)$. For $m=1$ (2.38) holds with equality sign for every $A$. For $m=2(2.38)$ holds, but the equality statement stated above does not fit this case.

REMARK 2. The only essential assumption about $A$ is that $|A|$ is symmetric. $|A| \in \mathscr{A}_{n}([s])$ includes the additional assumption that the components of $[s]$ are positive and distinct. This assumption is needed only to obtain the equality statement.

The second generalization deals with minors of matrices. We introduce now several concepts and notations.

Let $p$ and $n$ be natural numbers, $1 \leqq p \leqq n$. Denote

$$
Q_{p_{n}}=\left\{\left(i_{1}, \cdots, i_{p}\right) \mid 1 \leqq i_{1}<i_{2}<\cdots<i_{p} \leqq n\right\}
$$

$\left(i_{1}, \cdots, i_{p}\right.$ are natural numbers).

Let $i=\left(i_{1}, \cdots, i_{p}\right)$ and $j=\left(j_{1}, \cdots, j_{p}\right)$ be elements of $Q_{p_{n}}$, and let $A$ be a $n \times n$ matrix. The minor of $A$ formed from the rows $\left(i_{1}, \cdots, i_{p}\right)$ and the columns $\left(j_{1}, \cdots, j_{p}\right)$ is denoted by

$$
A\left(\begin{array}{c}
i_{1}, \cdots, i_{p} \\
j_{1}, \cdots, j_{p}
\end{array}\right)=A\left(\begin{array}{c}
i \\
j
\end{array}\right)
$$

The pth compound matrix of $A$ is denoted by $C_{p}(A) . C_{p}(A)$ is a $\left(\begin{array}{l}n \\ p\end{array}\right) \times\left(\begin{array}{l}n \\ p\end{array}\right)$ matrix with elements $A\left(\begin{array}{l}i \\ j\end{array}\right)$.

Let us now define the class of matrices $\left|\mathscr{A}_{n}([s])\right|$. A matrix $A$ belongs to the class $\left|\mathscr{A}_{n}([s])\right|$ if and only if $A$ is symmetric and $|A|$ belongs to $\mathscr{A}_{n}([s])$. Note that the definition includes the demand that all the components of $[s](A), A \in \mathscr{A}_{n}([s]) \mid$, are positive and distinct. Note also that a matrix belonging to $\left|\mathscr{A}_{n}([s])\right|$ can be complex.

In [6, formula 12] Schneider obtained the following result: Let $A$ be $a n \times n$ matrix and $p$ a natural number, $1 \leqq p \leqq n$. Then

$$
\sum_{j \in Q_{p_{n}}}\left|A\left(\begin{array}{c}
i \\
j
\end{array}\right)\right| \leqq\left[s_{i_{1}}\right] \cdots \cdot\left[s_{i_{p}}\right], i=\left(i_{1}, \cdots, i_{p}\right) .
$$

In [5] Ostrowski obtained the following equality statement: If $\left[s_{i_{1}}\right] \cdot \cdots \cdot\left[s_{i_{p}}\right] \neq 0$ then the equality sign in (2.41) holds if and only if in every column of the submatrix of $A$ formed from the $p$ rows $i_{1}, \cdots, i_{p}$, there exists at most one nonzero element. From this statement follows: If $A \in\left|\mathscr{A}_{n}([s])\right|$ and if $p \geqq 2$ then the equality sign in (2.41) holds for every $i \in Q_{p_{n}}$ if and only if $A$ is a diagonal matrix.

We bring now the second generalization of Theorem 2: Let $p$ and $n$ be natural numbers, $1 \leqq p \leqq n$. In the following two cases

(1) $m=3,4,5$ and $n=1,2, \cdots$

(2) $m=3,4, \cdots$ and $n=1,2,3$ 
the inequality

$$
\sum_{i, j \in Q_{p_{n}}}\left|A^{m}\left(\begin{array}{l}
i \\
j
\end{array}\right)\right| \leqq \sum_{i \in Q_{p_{n}}}\left(\left[s_{i_{1}}\right] \cdots \cdot\left[s_{i_{p}}\right]\right)^{m}
$$

holds for every $A$ belonging to $\left|\mathscr{A}_{n}([s])\right|$. The equality sign in these two cases holds if and only if $A$ is diagonal.

Proof. As $A$ is symmetric, the compound matrix $C_{p}(A)$ is also symmetric. Applying (2.38) to $C_{p}(A)$ (see Remark 2 after $(2.38)$ ), we obtain

$$
\begin{aligned}
S\left\{\left|\left[C_{p}(A)\right]^{m}\right|\right\} & =S\left[\left|C_{p}\left(A^{m}\right)\right|\right]=\sum_{i \in Q_{p_{n}}}\left|A^{m}\left(\begin{array}{l}
i \\
j
\end{array}\right)\right| \\
& \leqq \sum_{i \in Q_{p_{n}}}\left(\sum_{j \in Q_{p_{n}}}\left|A\left(\begin{array}{l}
i \\
j
\end{array}\right)\right|\right)^{m} .
\end{aligned}
$$

(2.42) follows from (2.41) and (2.43). For $p=1$ the equality statement follows from the equality statement corresponding to (2.38). Equality in (2.42) for $p \geqq 2$ implies equality in (2.41) for every $i \in Q_{p_{n}}$. As $A \in\left|\mathscr{A}_{n}([s])\right|$, it follows from the equality statement corresponding to (2.41) that $A$ is diagonal. It is obvious that if $A$ is diagonal then equality holds in (2.42).

Remark 1. For $p=1$ and $A \in \mathscr{A}_{n}(s)$ (2.42) reduces to $\left(2.4^{\prime}\right)$. (2.42), including the equality statement, holds for $p \geqq 2$ also for $m=1,2$.

REMARK 2. If the conjecture (2.4) stated at the beginning of this chapter holds true, then the two generalizations given in this section hold also for all $m$ and $n$.

The author wishes to thank Professor B. Schwarz for his guidance and help in the preparation of this paper.

\section{References}

1. D. Gale, The Theory of Linear Economic Models, McGraw-Hill, New-York, 1960.

2. G. H. Hardy, J. E. Littlewood and G. Pólya, Inequalities, 2nd edition, Cambridge, 1959.

3. D. London, Nonnegative matrices with stochastic powers, Israel J. Math. 2 (1964), 237-244.

4. M. Marcus and M. Newman, The sum of the elements of the powers of a matrix, Pacific J. Math. 12 (1962), 627-635.

5. A. M. Ostrowski, Note on a theorem by Hans Schneider, J. London Math. Soc. 37 (1962), 225-234.

6. H. Schneider, An inequality for latent roots applied for determinants with dominant principal diagonal, J. London Math. Soc. 28 (1953), 8-20.

Technion-Israel Institute of Technology, Haifa, IsRael 


\section{PACIFIC JOURNAL OF MATHEMATICS}

\section{EDITORS}

\author{
H. SAMmLSON \\ Stanford University \\ Stanford, California \\ R. M. BLUMENTHAL \\ University of Washington \\ Seattle, Washington 98105
}

\author{
*J. DUGUNDJI \\ University of Southern California \\ Los Angeles, California 90007
}

RICHARD ARENS

University of California

Los Angeles, California 90024

\section{E. F. BECKENBACH \\ B. H. NEUMANN \\ ASSOCIATE EDITORS}

\section{SUPPORTING INSTITUTIONS}

\author{
UNIVERSITY OF BRITISH COLUMBIA \\ CALIFORNIA INSTITUTE OF TECHNOLOGY \\ UNIVERSITY OF CALIFORNIA \\ MONTANA STATE UNIVERSITY \\ UNIVERSITY OF NEVADA \\ NEW MEXICO STATE UNIVERSITY \\ OREGON STATE UNIVERSITY \\ UNIVERSITY OF OREGON \\ OSAKA UNIVERSITY \\ UNIVERSITY OF SOUTHERN CALIFORNIA
}

\author{
STANFORD UNIVERSITY \\ UNIVERSITY OF TOKYO \\ UNIVERSITY OF UTAH \\ WASHINGTON STATE UNIVERSITY \\ UNIVERSITY OF WASHINGTON \\ * * * \\ AMERICAN MATHEMATICAL SOCIETY \\ CHEVRON RESEARCH CORPORATION \\ TRW SYSTEMS \\ NAVAL ORDNANCE TEST STATION
}

\footnotetext{
Mathematical papers intended for publication in the Pacific Journal of Mathematics should be typewritten (double spaced). The first paragraph or two must be capable of being used separately as a synopsis of the entire paper. It should not contain references to the bibliography. Manu. scripts may be sent to any one of the four editors. All other communications to the editors should be addressed to the managing editor, Richard Arens at the University of California, Los Angeles, California 90024.

50 reprints per author of each article are furnished free of charge; additional copies may be obtained at cost in multiples of 50 .
}

The Pacific Journal of Mathematics is published monthly. Effective with Volume 16 the price per volume (3 numbers) is $\$ 8.00$; single issues, $\$ 3.00$. Special price for current issues to individual faculty members of supporting institutions and to individual members of the American Mathematical Society: $\$ 4.00$ per volume; single issues $\$ 1.50$. Back numbers are available.

Subscriptions, orders for back numbers, and changes of address should be sent to Pacific Journal of Mathematics, 103 Highland Boulevard, Berkeley 8, California.

Printed at Kokusai Bunken Insatsusha (International Academic Printing Co., Ltd.), No. 6, 2-chome, Fujimi-cho, Chiyoda-ku, Tokyo, Japan.

PUBLISHED BY PACIFIC JOURNAL OF MATHEMATICS, A NON-PROFIT CORPORATION

The Supporting Institutions listed above contribute to the cost of publication of this Journal, but they are not owners or publishers and have no responsibility for its content or policies.

* Paul A. White, Acting Editor until J. Dugundji returns. 


\section{Pacific Journal of Mathematics}

\section{Vol. 16, No. $3 \quad$ BadMonth, 1966}

Gert Einar Torsten Almkvist, Stability of linear differential equations with

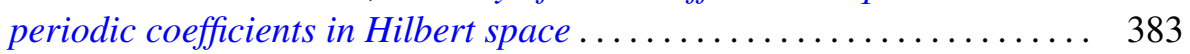

Richard Allen Askey and Stephen Wainger, A transplantation theorem for ultraspherical coefficients ................................ 393

Joseph Barback, Two notes on regressive isols .................. 407

Allen Richard Bernstein and Abraham Robinson, Solution of an invariant subspace problem of K. T. Smith and P. R. Halmos .............. 421

P. R. Halmos, Invariant subspaces of polynomially compact operators . . . . 433

Leon Bernstein, New infinite classes of periodic Jacobi-Perron algorithms.................................... 439

Richard Anthony Brualdi, Permanent of the direct product of matrices .... . 471

W. Wistar (William) Comfort and Kenneth Allen Ross, Pseudocompactness and uniform continuity in topological groups .................. 483

James Michael Gardner Fell, Algebras and fiber bundles . . . . . . . . . . . . 497

Alessandro Figà-Talamanca and Daniel Rider, A theorem of Littlewood and lacunary series for compact groups ..................... 505

David London, Two inequalities in nonnegative symmetric matrices...... 515

Norman Jay Pullman, Infinite products of substochastic matrices ........ 537

James McLean Sloss, Reflection and approximation by interpolation along

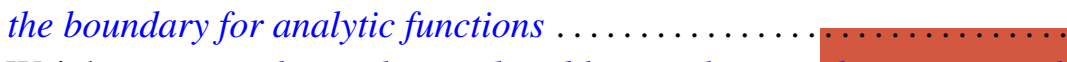

Carl Weinbaum, Visualizing the word problem, with an application to sixth groups................................... 